\title{
ANTIMICROBIAL EFFECTS OF OCIMUM SANCTUM ON ORAL MICROBES
}

\author{
AMRITA PARIDA ${ }^{*}$, SALMAN SIDDEEQH ${ }^{2}$, MAJI JOSE ${ }^{3}$, MANJU V ${ }^{4}$
}

${ }^{1}$ Department of Pharmacology, Kasturba Medical College, Mangalore, Manipal Academy of Higher Education, Manipal - 575 001, Karnataka, India. ${ }^{2}$ Department Oral Pathology and Microbiology, Century Dental College, Poinachi, Kerala, India. ${ }^{3}$ Department of Oral Pathology and Microbiology, Yenepoya Dental College, Mangalore, Karnataka, India. ${ }^{4}$ Department of Paediatrics, Melaka Manipal Medical College, Manipal Academy of Higher Education, Manipal - 576 104, Karnataka, India. Email: amrita_parida@yahoo.com

Received: 14 November 2017, Revised and Accepted: 05 February 2018

\section{ABSTRACT}

Objective: Ocimum sanctum (Tulsi) of the Lamiaceae family has been used since centuries for its medicinal properties. Tulsi powder has been utilized for cleaning tooth and is known to be effective in maintaining oral hygiene. Although the medical usefulness of tulsi is investigated extensively, its effectiveness in treating oral diseases is not yet established, and only a few studies are available in the literature regarding this aspect. Therefore, in the current study, the antimicrobial activity of alcoholic extract of tulsi on oral microbes was explored.

Methods: Ethanolic extract was obtained from the leaves of $O$. sanctum (tulsi) using the Soxhlet apparatus. The concentration of $400 \mu \mathrm{g} / \mathrm{ml}$ was chosen to test the antimicrobial properties against common oral microbes such as Prevotella intermedia, Streptococcus mutans, Candida albicans, Lactobacillus acidophilus, Streptococcus mitis, and Peptostreptococcus using agar well diffusion and broth dilution method. Chlorhexidine was a positive control. To compare and correlate the results, Mann-Whitney U-test was applied.

Results: The ethanolic extract at a concentration of $400 \mu \mathrm{g} / \mathrm{ml}$ showed an inhibitory effect against Streptococcus and Streptococcus with an inhibitory zone of $7.33 \mathrm{~mm}$ each. The extract was found to be effective against $P$. intermedia and Peptostreptococcus. It was also effective against candida (zone of inhibition was $10.67 \mathrm{~mm}$ ). However, no inhibitory effect was seen on Lactobacillus.

Conclusion: $O$. sanctum extract was found to have inhibitory effects on oral microbes, and hence, pharmaceutical formulations prepared from tulsi extracts could have a potential to be used as a part of routine dental hygiene.

Keywords: Pharmacognosy, Plant extracts, Antimicrobial, Basil, Herbal remedies

(C) 2018 The Authors. Published by Innovare Academic Sciences Pvt Ltd. This is an open access article under the CC BY license (http://creativecommons. org/licenses/by/4. 0/) DOI: http://dx.doi.org/10.22159/ajpcr.2018.v11i5.23623

\section{INTRODUCTION}

Ocimum sanctum (Tulsi) is well known for its religious, medicinal, and culinary usefulness. It is routinely used in Ayurveda for diseases such as diabetes, bronchitis, dermal conditions, and arthritis [1]. Although the effectiveness of tulsi in all these ailments has been extensively investigated, its role in routine oral hygiene is less studied. There are only limited studies done in this aspect [1-4]. Hence, against this background, the antimicrobial activity of the crude ethanolic extract of tulsi on microbes commonly producing dental caries and periodontitis was investigated. This study would help to verify the claimed validity of traditional indigenous practice, and identification of beneficial effects if any may be incorporated into modern oral care system to make it accessible to the community at large.

\section{METHODS [5]}

\section{Preparation of ethanolic extract [6]}

Institutional Ethics Committee clearance was obtained before the start of the study. Study duration was of 1 year. Leaves of 0 . sanctum were collected and then validated by a botanist. The leaves were then carefully cleaned with distilled water and then air dried for 5 days. It was then powdered using an electric blender. Soxhlet apparatus was used for the extraction process using $250 \mathrm{~mL}$ of absolute ethanol. $50 \mathrm{~g}$ of the powder was put into the apparatus. The filtrate was concentrated with the help of a rotary evaporator. The extract obtained was stored at $4{ }^{\circ} \mathrm{C}$ in airtight containers till further use. $50 \mathrm{~g}$ of dried leaves gave a yield of $9.3 \mathrm{~g}$. Then, the extract was dissolved in dimethyl sulfoxide (DMSO) to prepare a concentration of $400 \mu \mathrm{g} / \mathrm{ml}$ which was used for the study.

\section{Antimicrobial property assessment}

Test organisms' collection and isolation

The microbes for the study were obtained from patients who had come to the Yenepoya Dental College outpatient department for treatment. The patients gave their informed consent before the start of the procedure. Cariogenic organisms were collected from tooth caries scrapings while the periodontal organisms were obtained from periodontal pockets. The organisms were transported in Brain heart infusion (BHI) broth.

The samples were then placed in an anaerobic chamber/incubator and kept for incubation for $48 \mathrm{~h}$. Then, manually plating on different media such as chocolate agar, sheep blood agar, and sabouraud dextrose agar was done. Strict aseptic precautions were maintained, and the process was carried out in a laminar airflow chamber. These plates were incubated for $24 \mathrm{~h}$. After incubation, the culture plates were observed to look for the formation of any microbial colonies. The colonies were identified by standard microbiological tests.

The organisms were preserved in BHI and Robertson's Cooked Meat broth. Subcultures were done serially for every $72 \mathrm{~h}$ for the entire study period. A check on the purity of broth cultures was maintained from time to time using standard techniques [5]. Organisms studied were Streptococcus mutans, Streptococcus mitis, Lactobacilli, Peptostreptococcus, Prevotella intermedia, and Candida albicans.

\section{Preparation of inoculum [7]}

The organisms blended in BHI broth were further incubated for $24 \mathrm{~h}$ at $37^{\circ} \mathrm{C}$. These suspensions were then diluted using BHI broth until turbidity equivalent to McFarland 0.5 standard $5 \times 10^{5}$ colony forming units $/ \mathrm{ml}$ was achieved. They were used as inoculum for assessing the antimicrobial activity of the tulsi extract.

Antimicrobial activity screening of the ethanolic extract [8] Agar well diffusion method was used to screen for the antimicrobial property of the tulsi extract. Lino and Deogracious technique with minor modifications was adopted by us. The freshly made inoculum 
was swabbed on the Mueller Hinton agar plate with the help of a sterile cotton swab. With the help of a cork-borer, five wells were made on the media. Each well was $6 \mathrm{~mm}$ in diameter. Three of these labeled wells were then filled with $50 \mu \mathrm{l}$ of the tulsi extract. The other two wells were filled with same volumes of positive (chlorhexidine) and negative control (DMSO), respectively. The contents were allowed to diffuse into the media, and then the plates were incubated $\left(37^{\circ} \mathrm{C}\right.$ for $\left.24 \mathrm{~h}\right)$. Then, the zone of inhibition was noted

Different dilutions of the extract which showed antimicrobial activity in the agar well diffusion were taken, and the minimum inhibitory concentration (MIC) noted. Minimum bactericidal concentration (MBC) was determined by the standard procedures [5]

\section{Statistical analysis}

Mann-Whitney U-test was performed on the results obtained. Statistical analysis was completed using SPSS software version 15. Level of significance was set at $P<0.05$

\section{RESULTS}

The current study evaluated the antimicrobial properties of ethanolic extract of $O$. sanctum. The zone of inhibition, produced by $400 \mu \mathrm{g} / \mathrm{L}$ ethanolic extract of $O$. sanctum was analyzed and compared with chlorhexidine, the positive control.

The extract showed a zone of inhibition of $6 \mathrm{~mm}$ and $7.33 \mathrm{~mm}$ against Prevotella and $S$. mitis, respectively. It was also seen to be active against $C$. albicans (10.67 $\mathrm{mm})$, Peptostreptococcus $(8.67 \mathrm{~mm})$, and S. mitis $(7.33 \mathrm{~mm})$. However, no antibacterial activity was seen against Lactobacillus. The zone of inhibition with Chlorhexidine was $21.33 \mathrm{~mm}$ for all of the above organisms except Prevotella $(10 \mathrm{~mm})$. The zone of inhibition for Peptostreptococcus was statistically similar for tulsi and chlorhexidine.

In this study, the range of MIC was from $25 \mu \mathrm{g} / \mathrm{ml}$ to $400 \mu \mathrm{g} / \mathrm{ml}$. MIC and $\mathrm{MBC}$ of the $O$. sanctum extract against various pathogens are depicted in Table 1.

\section{DISCUSSION}

Tulsi plant has been extensively harnessed by the practitioners of traditional medicine for its immense usefulness in various ailments. It holds a plethora of medicinally important compounds. Different parts of the tulsi plant have been put to use in treating patients. It is found to be effective for diseases ranging from simple diarrhea to complicated conditions like arthritis. It is believed to have protective effects on heart and liver and also found to have anticancer properties [9]. The exact chemical that is responsible for the beneficial effects of tulsi is still elusive. It is probably the complex interaction between the numerous chemicals present in the herb that finally determines its biological activity. The proportions of the composition of these active compounds in tulsi vary from plant to plant and are not constant. The active principles may also be affected by the harvesting, processing, and storage conditions of the plant products. A large number of phytochemicals has been isolated from the tulsi leaves which include eugenol, euginal, urosolic acid, and carvacrol.

Table 1: The MIC and MBC of the alcoholic extract of $O$. sanctum against different microbes

\begin{tabular}{lll}
\hline Test organisms & MIC & MBC \\
\hline S. mutans & 25 & 25 \\
S. mitis & 400 & 800 \\
Lactobacillus & 000 & 000 \\
C. albicans & 000 & 000 \\
Peptostreptococcus & 25 & 25 \\
Prevotella & 400 & 800 \\
\hline
\end{tabular}

MIC: Minimum inhibitory concentration, MBC: Minimum bactericidal

concentration, S. mutans: Streptococcus mutans, S. mitis: Streptococcus mitis,

C. albicans: Candida albican
Variety of other compounds including saponins, phenols, tannins, and flavonoids has also been isolated [9]. Among all of these active principles, eugenol (chemically known as 1-hydroxy-2-methoxy-4-allylbenzene), are believed to be responsible for the antimicrobial effects [10]. Some authors also suggest that the antimicrobial effect is due to linoleic acid which was seen to be effective against Bacillus pumilus, Staphylococcus aureus, and Pseudomonas aeruginosa [11]. However, because of the chemical complexity of the constituents of tulsi, it is difficult to standardize tulsi extract and duplicate its effects in the laboratory.

After a thorough search in the literature, only a few studies could be identified in which the efficacy ethanolic extract of tulsi leaves as an antimicrobial agent on the oral pathogens was evaluated. In one of such studies by Agarwal et al., 15 different concentrations of 0 . sanctum extracts were tested, and they noted a zone of inhibition of $22 \mathrm{~mm}$, against $S$. mutans at concentration $4 \% \mathrm{w} / \mathrm{v}$ of the 0 . sanctum extract [1]. In another study, [2] $10 \%$ of alcoholic extract showed an inhibitory effect against $S$. mutans, $S$. mitis, $S$. sanguis, and Lactobacillus. Although this study showed the efficacy of tulsi against Lactobacillus, we did not find any inhibitory effects. In a similar study by Mallikarjun et al., $5 \% \mathrm{w} / \mathrm{v}$ and $10 \% \mathrm{w} / \mathrm{v}$ concentration of alcoholic extract of tulsi was found to have inhibitory action against the periodontal pathogen, Aggregatibacter actinomycetemcomitans but ineffective against Porphyromonas gingivalis and P. intermedia [4]. However, in contrast, we found the extract to be effective against $P$. intermedia. These differences in the result could be due to the variations in the concentration of the extract used. The other reason could be due to change in the chemical composition of the active principle because of the variations in the strains of the plants as mentioned earlier. Antimicrobial activity of $6 \% \mathrm{w} / \mathrm{v}$ alcoholic extract of tulsi extract against A. actinomycetemcomitans was also seen in a study conducted by Eswar et al., None of these studies have observed the effect of tulsi extract on Candida whereas we found tulsi to have an effective antimicrobial activity on Candida.

Sometimes, the zone of inhibition observed in the agar medium may not be an actual reflection of the antimicrobial activity of the compound. This is because numerous factors affect the antimicrobial activity of the plant extracts. Variations in the diffusion of the active principle through the agar media could be one of the reasons [8]. Hence, determination of the MIC and MBC was also taken into consideration in our study.

\section{Limitation of the study}

The major limitation is that we have performed the study on crude extract and isolation of the active principle was not within the scope of our study. Further, the exact mechanism by which tulsi exerts its antibacterial effects cannot be commented based on our study.

\section{CONCLUSION}

In our study, we found that the alcoholic extract of 0 . sanctum has antimicrobial activity against oral microbes and may be useful as an adjunct to other products involved in the maintenance of oral hygiene. However, further studies to find out the adverse effects and long-term toxicities are required before this extract can be put to daily use. The antimicrobial activity against other microorganisms and synergistic action along with various antimicrobials should also be investigated.

\section{ACKNOWLEDGMENT}

We would like to thank Dr. Vidya Pai, Professor, and Head, Department of Microbiology, Yenepoya University, Mangalore, Karnataka, for her support and guidance during the study.

\section{AUTHOR'S CONTRIBUTIONS}

All the four authors were involved in the execution of research, analysis of data, writing, and editing of the manuscript.

\section{CONFLICTS OF INTEREST}

None. 


\section{REFERENCES}

1. Agarwal P, Nagesh L, Murlikrishnan. Evaluation of the antimicrobial activity of various concentrations of Tulsi (Ocimum sanctum) extracts against Streptococcus mutans: An in vitro study. Indian J Dent Res 2010;21:357-9.

2. Pai RK, Bhat SS, Salman A, Chandra J. Use of an extract of Indian sacred plant Ocimum sanctum as an anticariogenic agent: An in vitro study. Int J Clin Pediatr Dent 2015;8:99-101.

3. Eswar P, Devaraj CG, Agarwal P. Anti-microbial activity of tulsi (Ocimum sanctum [Linn.]) extract on a periodontal pathogen in human dental plaque: An in vitro study. J Clin Diagn Res 2016;10:53-6.

4. Mallikarjun S, Rao A, Rajesh G, Shenoy R, Pai M. Antimicrobial efficacy of Tulsi leaf (Ocimum sanctum) extract on periodontal pathogens: An in vitro study. J Indian Soc Periodontol 2016;20:145-50.

5. Siddeeqh S, Parida A, Jose M, Pai V. Estimation of antimicrobial properties of aqueous and alcoholic extracts of Salvadora persica (miswak) on oral microbial pathogens-an in vitro study. J Clin Diagn
Res 2016;10:13-6.

6. Al-Bayati FA, Sulaiman KD. In the vitro antimicrobial activity of Salvadora persica $\mathrm{L}$. extracts against some isolated oral pathogens in Iraq. Turkish J Biol 2008;32:57-62.

7. Koneman EW, Allen SD, Janda WM, Schreckenberger PC. Color Atlas and Textbook of Diagnostic Microbiology. Philadelphia: Lippicott Williams \& Wilkins; 1997. p. 491-537.

8. Cyriac MB, Pai V, Varghese I, Shantaram MJ. Antimicrobial properties of Areca catechu (areca nut) husk extracts against common oral pathogens. IJRAP 2012;3:81-4

9. Pattanayak P, Behera P, Das D, Panda SK. Ocimum sanctum Linn. A reservoir plant for therapeutic applications: An overview. Pharmacogn Rev 2010;4:95-105.

10. Prakash P, Gupta N. Therapeutic uses of Ocimum sanctum Linn. (Tulsi) with a note on eugenol and its pharmacological actions: A short review. Indian J Physiol Pharmacol 2005;49:125-31.

11. Singh S, Malhotra M, Majumdar DK. Antibacterial activity of Ocimum sanctum L.fixed oil. Indian J Exp Biol 2005;43:835-7. 